\title{
Captura, acondicionamiento y primer desove de sargo Anisotremus scapularis en la Región Tacna
}

\author{
Capture, conditioning and first spawning of Anisotremus scapularis sargo in the \\ Tacna Region
}

${ }^{1}$ Luis Antonio Espinoza Ramos

ORCID: 0000-0001-7958-7331

${ }^{2}$ Víctor Franco Chili Layme

${ }^{3}$ Renzo Gerardo Pepe Victoriano

ORCID: 0000-0003-1483-4164

${ }^{4}$ Jorge Pino Choqueapaza

${ }^{5}$ Zumilda Contreras Mamani

ORCID: 0000-0002-7630-1411

ORCID: 0000-0002-0967-5528

ORCID: 0000-0002-2654-9996

\section{RESUMEN}

La presente investigación tuvo como objetivo desarrollar un sistema de captura para acondicionamiento de reproductores de sargo en el Centro de Acuicultura Morro Sama- Tacna. Asimismo, desarrollar una técnica de alta efectividad, medida en sobrevivencia para la captura de ejemplares de sargo, en la Región Tacna. Además de describir los resultados sobre la captura acondicionamiento y reproducción de sargo Anisotremus scapularis. Para esto, se recolectaron 15 peces silvestres, los cuales al final del período de acondicionamiento alcanzaron el estado de maduración gonadal y desovaron en forma espontánea. Los 15 ejemplares de sargos Anisotremus scapularis fueron capturados con anzuelos en las playas de Llostay (Tacna), en el año 2016. Y fueron acondicionados en un tanque de 4 metros de diámetro con un sistema abierto de agua de mar, alimentados con muy muy Emerita analoga y alimento balanceado de formulación propia. Entre los resultados se encuentra una sobrevivencia de 73.33 \% (11 sargos) luego de 7 meses de acondicionamiento. El primer desove fue el 29 de octubre del 2016 a una temperatura de $18^{\circ} \mathrm{C}$.

Palabras clave: Acondicionamiento, reproduccion, sargo.

\begin{abstract}
The objective of this research was to develop a capture system for conditioning sargo breeders at the Morro SamaTacna Aquaculture Center. Likewise, to develop a highly effective technique, measured in survival for the capture of specimens of sargo, in the Tacna Region. Also, to describe the results on the capture conditioning and reproduction of Anisotremus scapularis. For this, 15 wild fish were collected, which at the end of the conditioning period reached the state of gonadal maturation and spawned spontaneously. The 15 specimens of Anisotremus scapularis sargos were caught with hooks on the beaches of Llostay (Tacna), in 2016. And they were conditioned in a 4 meter diameter tank with an open seawater system, fed with Emerita Analog and balanced feed of own formulation. It was reported a survival of $73.33 \%$ (11 sargos) after 7 months of conditioning. The first spawning was on October 29, 2016 at a temperature of $18^{\circ} \mathrm{C}$.
\end{abstract}

Keywords: Conditioning, reproduction, sargo.

${ }^{1}$ Facultad de Ciencias Agropecuarias. Universidad Nacional Jorge Basadre Grohmann. Tacna-Perú. E-mail: laer 54@hotmail.com ${ }^{2}$ Centro de Acuicultura Morro Sama. Fondo Nacional de Desarrollo Pesquero. Tacna- Perú. E-mail: victorchili@hotmail.com ${ }^{3}$ Facultad de Recursos Naturales Renovables. Universidad Arturo Prat. Arica-Chile. E-mail: rpepev@unap.cl, regepevic@gmail.com ${ }^{4}$ Centro de Acuicultura Morro Sama. Fondo Nacional de Desarrollo Pesquero. Tacna-Perú. E-mail: jorgepinochoqueapaza@hotmail.com ${ }^{5}$ Facultad de Ciencias Agropecuarias. Universidad Nacional Jorge Basadre Grohmann. Tacna-Perú. E-mail: czumilda@gmail.com 


\section{INTRODUCCIÓN}

La acuicultura es el cultivo de organismos acuáticos, tanto en zonas costeras como del interior, que implica intervenciones en el proceso de cría para aumentar la producción. Además, es el sector de producción de alimentos de más rápido crecimiento y representa ahora el $50 \%$ del pescado destinado a la alimentación a nivel mundial (FAO, 2014).

La acuicultura es el cultivo de organismos acuáticos, incluyendo peces, moluscos, crustáceos y plantas (algas) para consumo humano (Coll, 2016).

La acuicultura marina implica la producción comercial de especies, y tales lugares deben ser accesibles, libres de especies depredadoras y tener altos estándares de calidad del agua (Lloyd et al., 1991).

La producción de la maricultura presenta en los últimos años un vertiginoso avance, fundamentado en los progresos científicos y técnicos que han permitido conocer, con mayor exactitud y precisión, los procesos biológicos de la reproducción, la alimentación y el crecimiento de los animales marinos (González, 2011).

Un reto de la acuicultura es la diversificación y la reproducción controlada de nuevas especies de interés comercial. La reproducción de los peces es un proceso de marcado carácter rítmico, que se encuentra regulado por factores ambientales y otros como el fotoperiodo y la temperatura (Carrillo et al, 2009).

Sargo es una especie del Pacífico oriental y su hábitat se encuentra desde el sur de Colombia hasta Chile; del mismo modo, en las islas Galápagos y Malpelo (Tavera,2006).

El género Haemulon (familia Haemulidae) representa un excelente grupo en los Trópicos del mundo (Gilbert, 2005). Contiene 19 especies nominales distribuidas en todo el Pacífico oriental tropical y el Atlántico occidental (Rocha et al., 2008).

Además, Sargo es una de las seis especies del género registrada para el Perú. Entre sus características encontramos que es de color gris plateado con una mancha negra, tanto en la axila de la aleta pectoral como la base del ultimo radio dorsal y anal. (Chirichigno, 1974). Asimismo, es un pez bento-pelágico carnívoro que forma grandes cardúmenes (Allen et al., 2010).

Otra característica es que vive en zonas rocosas y sustratos duros con fuertes corrientes o mareas y a profundidades de $35 \mathrm{~m}$ (Carrillo, 2009).

En Perú, el cultivo de peces marinos nativos está orientado principalmente a la investigación, donde se ha capturado reproductores y juveniles silvestres de Paralichthys adspersus (lenguado peruano) (Chili et al., 2009); además de haber importado adultos de Scophthalmus maximus (turbot) (Pauro, 2007).

Hay que tener en cuenta, que es indispensable contar con ejemplares silvestres bien adaptados a la reproducción en cautiverio, para obtener progenies de buena calidad (Gallardo, 2016).

El objetivo del presente estudio fue desarrollar un sistema de captura para acondicionamiento de reproductores de sargo en el Centro de Acuicultura Morro Sama- Tacna. Además, de desarrollar una técnica de alta efectividad, medida en sobrevivencia para la captura de ejemplares de sargo, en la Región Tacna. Establecer una técnica de alimentación en cautiverio para los reproductores recién acondicionados. Otro de los objetivos fue conocer el porcentaje de viabilidad de las ovas.

\section{MATERIAL Y MÉTODOS}

\section{Captura de reproductores}

Con el propósito de obtener ejemplares silvestres de Sargo Anisotremus scapularis, se realizaron 3 campañas de pesca el 11 y 17 de marzo y el 6 de abril de 2016.

La pesca de los especímenes se realizó en la zona de rocosa marina de la playa Llostay (Perú) (18 $\left.10^{\circ} 26^{\prime \prime} \mathrm{S}, 70^{\circ} 38^{\prime} 37^{\prime \prime O}\right)$, a $50 \mathrm{~m}$ desde la línea de baja marea hacía el mar (Figura 1).

El aparejo empleado para la captura de los ejemplares consistió en anzuelos con carnada de muy muy de Emerita analoga .

Los sargos fueron trasladados a la orilla de forma inmediata y la eliminación de anzuelos fue llevada a cabo por los mismos pescadores. Luego, se procedió a la reanimación de los ejemplares en una tina oval de $50 \mathrm{~L}$, mediante chorros de agua de mar batida utilizando un balde de 20 Ly jarras de 2 
L. Finalmente, se procedió a la evaluación física por los profesionales de campo, y a la selección de los ejemplares.

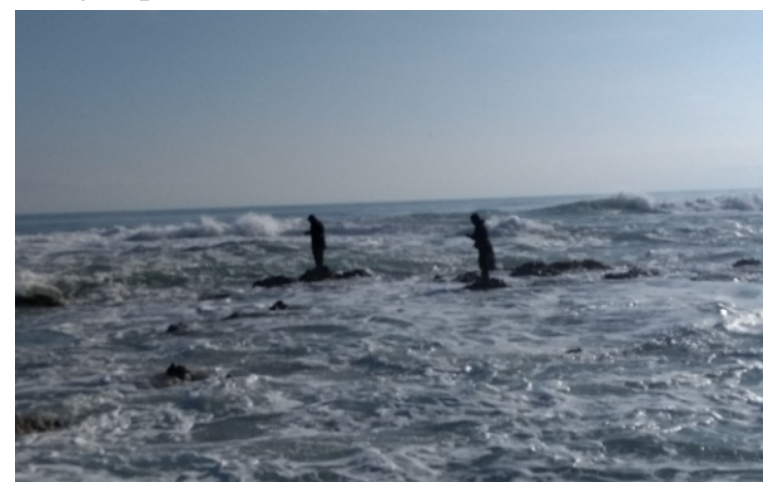

Figura 1. Captura de sargo.

\section{Transporte de Reproductores}

Se procedió al traslado al centro acuícola de Morro Sama (17 59'39.7"S, 7052'59.1"O). El traslado se efectuó en un tanque cúbico de $1000 \mathrm{~L}$ de capacidad llenado hasta los $500 \mathrm{~L}$ de agua marina cruda, instalado en una camioneta $4 \times 4$, en donde se suministró oxígeno, alimentado por un tanque conducido por una manguera de silicona y finalmente por una piedra difusora. La temperatura fue regulada durante la selección y transporte de ejemplares por medio de bolsas de 1 $\mathrm{kg}$ con hielo introducidas al tanque de transporte, la temperatura se mantuvo entre $19^{\circ} \mathrm{C}$ y $20^{\circ} \mathrm{C}$.

En el centro acuícola se aplicó el tratamiento preventivo con oxitetraciclina $99 \%$ a una concentración 50 ppm durante 60 minutos, en el mismo tanque de transporte.

\section{Acondicionamiento}

La etapa de acondicionamiento se llevó a cabo durante 7 días, durante los cuales se procedió a tratamientos parasitarios preventivos, mediante formol al $37 \%$ de pureza y a una relación de $1 \mathrm{ml}$ de formol por $6000 \mathrm{ml}$ de agua (1/6000 o 23.6 ppm).

Los tratamientos se efectuaron en un tanque de 4 m de diámetro y $9000 \mathrm{~L}$ de capacidad de volumen de agua, llenado hasta los $6000 \mathrm{~L}$. El caudal de agua fue de $0.61 \mathrm{~L} / \mathrm{s}$, además que el tanque estaba cubierto con dos capas de malla raschel, la cual permitía una intensidad de luz promedio de 335.33 Klux, con Luxometro, marca Hanna, en el nivel de agua (Figura 2).

La limpieza de tanque se realizó vaciando el tanque, por medio de barridos con escobas. La forma de barrido era en sentido radial, desde el borde interior del tanque hacia el centro donde se ubicaba la zona de desagüe. Esta actividad se realizaba en horas de la mañana $(8 \mathrm{am})$ y dos veces por semana (martes y viernes).

Los parámetros fisicoquímicos fueron la temperatura del agua y oxígeno disuelto, los cuales se registraron a las 6:00 am, 12:00 pm 6:00 pm y 12:00 am.

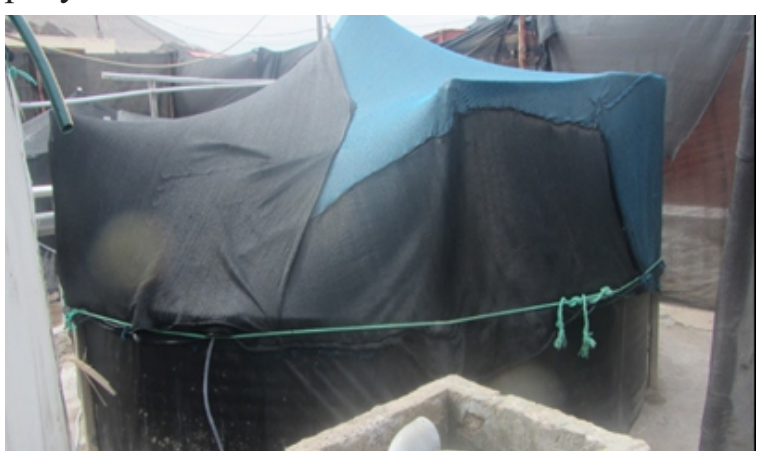

Figura 2. Tanque de cultivo.

\section{Alimentación}

$\mathrm{Al}$ inicio del acondicionamiento, se mantuvieron en ayunas durante 7 días, para luego seguir con la siguiente secuencia:

DIETA 1. Para lo cual se procedió a suministrarle el alimento vivo correspondiente muy muy Emerita analoga, observándose en el fondo del tanque restos de excretas con residuos de muy muy de color naranja.

DIETA 2. Se les dio muy muy Emerita analoga reposado con harina de pescado por unos 15 minutos.

DIETA 3. Se les dio alimento balanceado elaborado en forma artesanal (Figura 3) (Tabla 2).

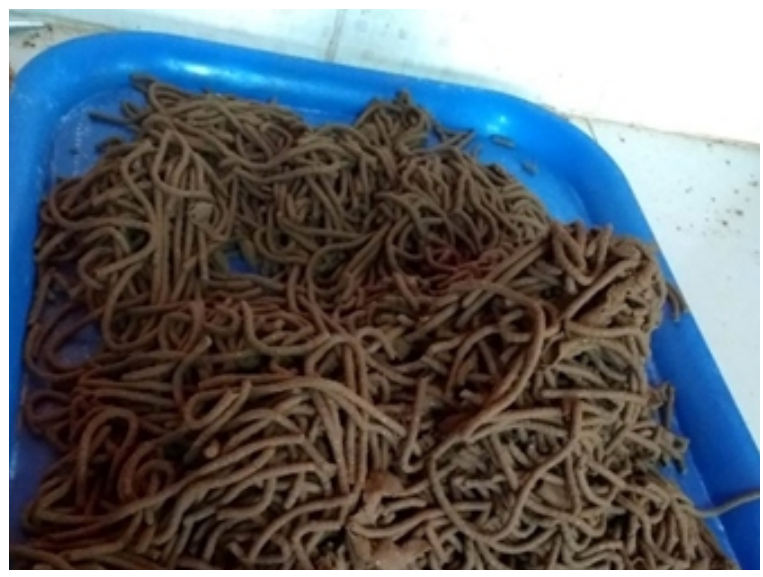

Figura 3. Alimento balanceado. 


\section{Implante de Chip}

El 04 de octubre de 2016 se efectuó el implante de un chip de identificación individual, en la zona dorsal intramuscular de los reproductores adultos, previamente anestesiados con eugenol, con la finalidad de tener su registro y seguimiento individual del crecimiento y el desove (Figura 4).

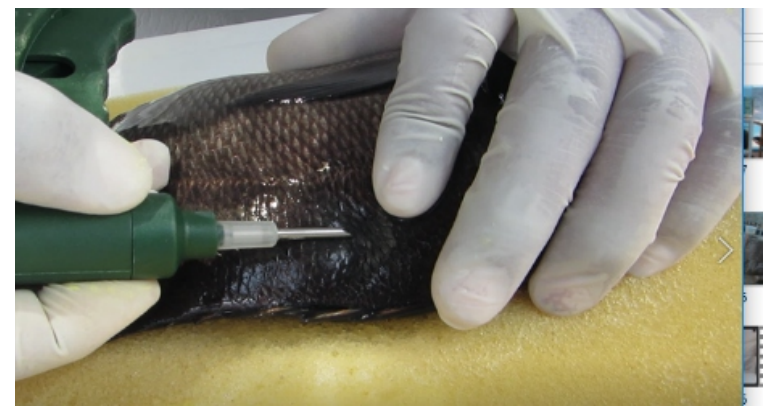

Figura 4. Implante de Chip.

\section{Primer desove}

La reproducción de Anisotremus scapularis se dio de manera espontánea en el tanque de cultivo, aproximadamente de 5:00 a 7:00 pm, la temperatura del agua de cultivo fue $18.0^{\circ} \mathrm{C}$.

Se realizó la colecta de ovas con un tamiz colector de $300 \mu \mathrm{m}$, a las 8:00 am. Y fueron concentradas $\mathrm{y}$ depositadas en un balde para su traslado a la sala de incubación, las ovas fueron desinfectadas con aquayodo $2 \mathrm{ml} / \mathrm{L}$ - ${ }^{1}$ de agua de mar.

Se evaluó la calidad de ovas mediante la medición de su diámetro, homogeneidad de tamaño y esfericidad (Figura 5).

La incubación se realizó en un tanque de $500 \mathrm{~L}$ de capacidad con volumen de agua útil de $400 \mathrm{~L}$, suministrado con aireación moderada a través de una piedra difusora de poro fino.

El número total de huevos fue cuantificado volumétricamente.

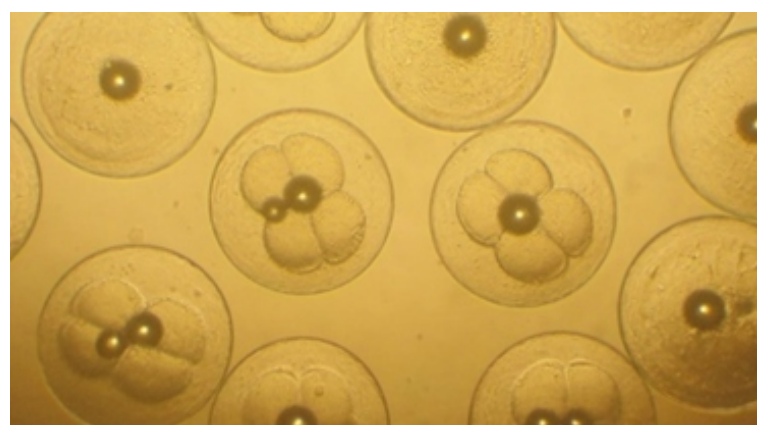

Figura 5. Ovas de A. scapularis.

\section{RESULTADOS}

\section{Captura y sobrevivencia}

Tabla 1. Captura y porcentajes de sobrevivencia de reproductores silvestres de Anisotremus scapularis por campaña de pesca.

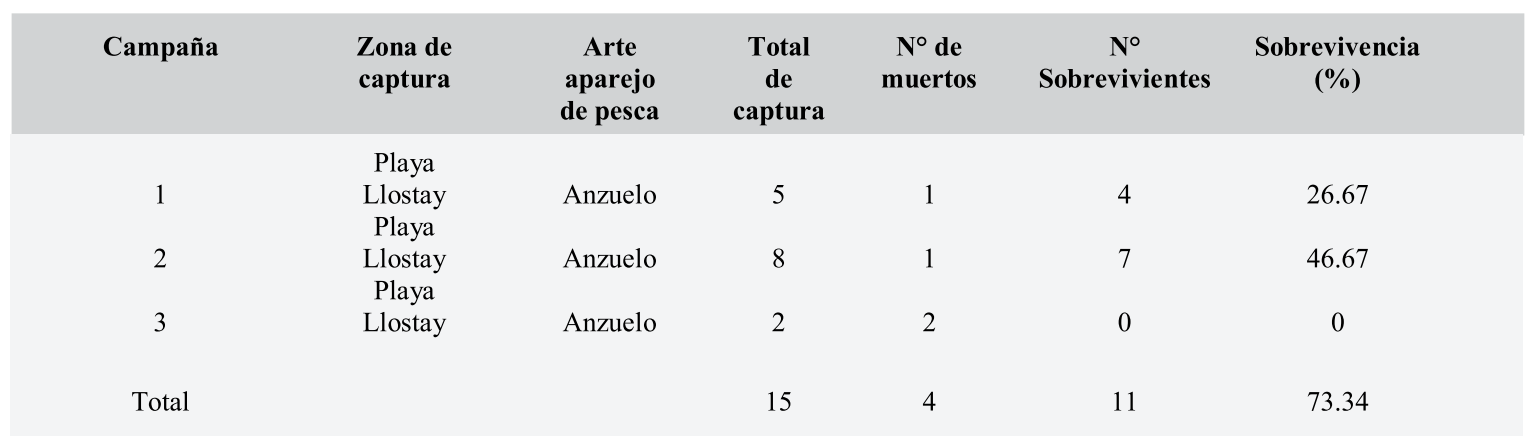

Se tiene una sobrevivencia del $73.34 \%$ de la captura y acondicionamiento de sargo.

\section{Acondicionamiento de reproductores}

La temperatura del agua se mantuvo entre un mínimo de $16.47{ }^{\circ} \mathrm{C}$ y un máximo de $20.10{ }^{\circ} \mathrm{C}$.
Mientras que la temperatura de ambiente tuvo un mínimo $18.82{ }^{\circ} \mathrm{C}$ y un máximo de $24.64{ }^{\circ} \mathrm{C}$ (Figura 6) . 


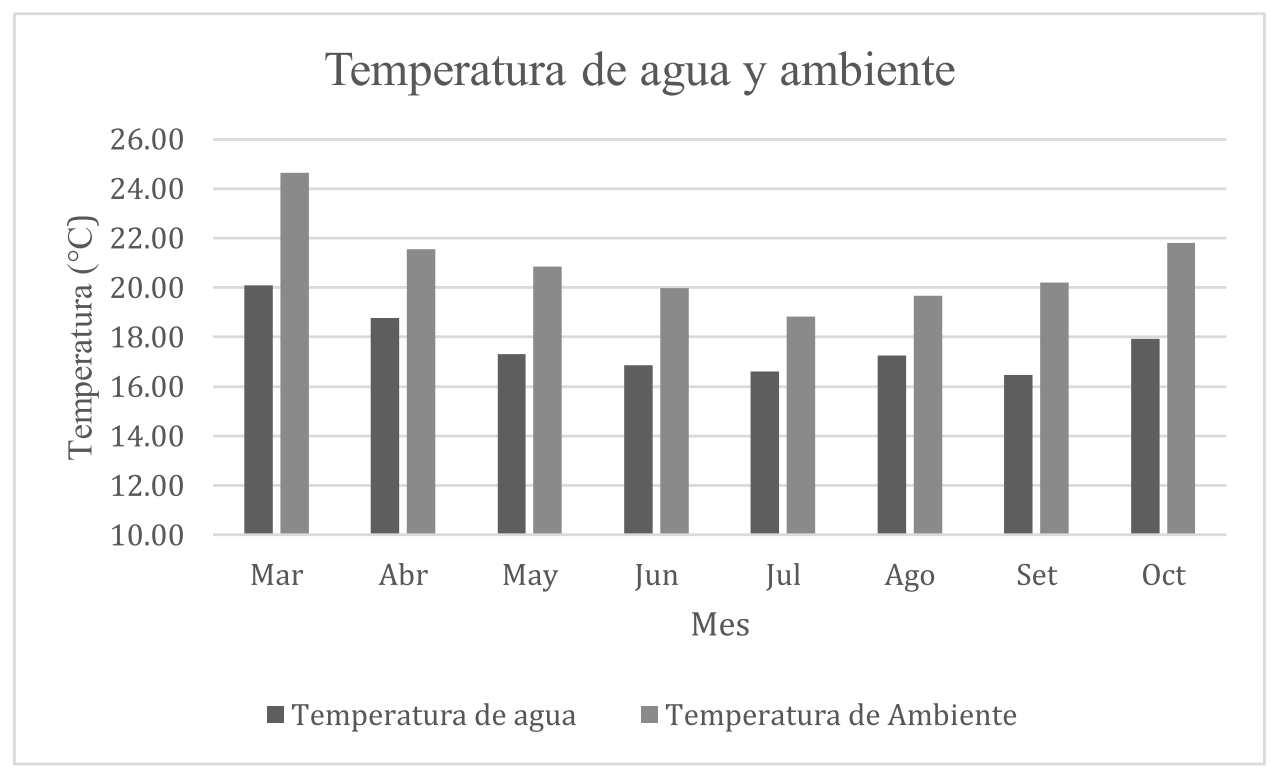

Figura 6. Temperatura registrada del agua y ambiente.

El oxígeno disuelto en el agua se mantuvo entre un mínimo $7.17 \mathrm{mg} \mathrm{L}^{-1} \mathrm{y}$ un máximo de $7.86 \mathrm{mg} \mathrm{L}^{-1}$ (Figura 7).

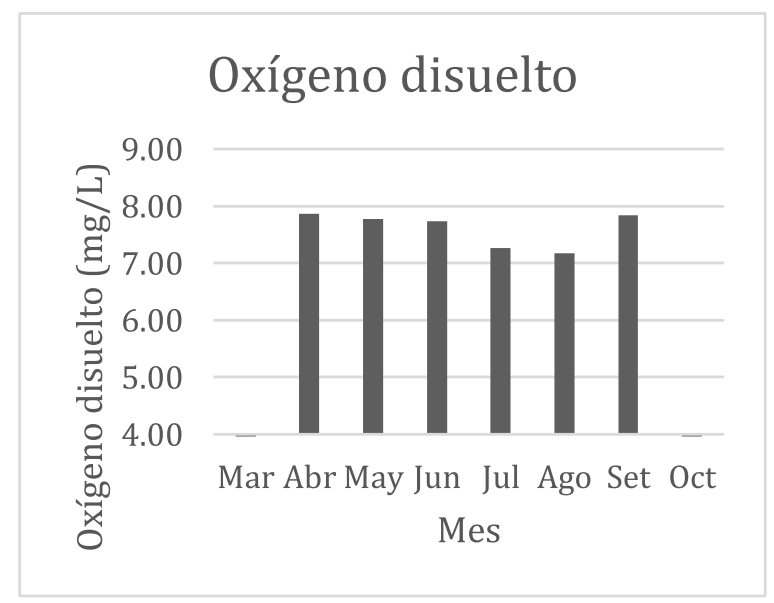

Figura 7. Oxígeno disuelto en el agua.

\section{Adaptación al alimento balaceado}

Tabla 2. Alimento balanceado para Anisotremus scapularis. Pellet húmedo.

\begin{tabular}{|lc|}
\hline Dieta & Porcentaje (\%) \\
\hline Proteina & 37.13 \\
Grasa & 6.56 \\
Ceniza & 9.45 \\
Fibra & 0.73 \\
Humedad & 44.32 \\
\hline
\end{tabular}

Se les proporciono una dieta de 37.13 de proteína.

\section{Identificación digital de los animales}

Tabla 3. Código de chip en cada una de los individuos.

\begin{tabular}{|ccr|}
\hline Código de Chip & Peso (kg) & Talla (cm) \\
\hline 939000001419265 & 2.293 & 44.70 \\
939000001418824 & 2.020 & 44.40 \\
939000001426446 & 1.629 & 41.40 \\
939000001425972 & 1.417 & 38.30 \\
939000001418802 & 1.192 & 38.50 \\
939000001419573 & 1.169 & 37.60 \\
939000001426408 & 0.859 & 35.00 \\
939000001426567 & 0.847 & 34.20 \\
939000001426218 & 0.833 & 32.00 \\
939000001418657 & 0.683 & 31.00 \\
939000001425610 & 0.598 & 30.00 \\
\hline
\end{tabular}

Se tienen sargos de una talla de $44.70 \mathrm{~cm}$ a 30.00 $\mathrm{cm}$.

\section{Desove}

\begin{tabular}{|c|c|c|c|c|c|}
\hline Fecha & $\begin{array}{c}\text { Ovas } \\
\text { viables } \\
\left({ }^{*} 1000\right)\end{array}$ & $\begin{array}{c}\text { Viabilidad } \\
\text { (\%) }\end{array}$ & Calidad & $\begin{array}{c}\text { Diámetro } \\
(\mu \mathrm{m})\end{array}$ & $\begin{array}{c}\text { Eclosión } \\
(\%)\end{array}$ \\
\hline 29-Oct & 59.4 & 79.1 & Buena & 783.13 & 93.2 \\
\hline
\end{tabular}

El tamaño de las ovas es de $783.13 \mu$. 


\section{DISCUSIÓN}

De acuerdo con Muñoz et al, (2012), para iniciar el cultivo de una especie, es importante disponer de un lote de reproductores que deben ser capturados del ambiente natural y ser acondicionados a cautiverio. Esto permitirá realizar estudios sobre su comportamiento, manejo, aceptación de alimento, sobrevivencia y crecimiento en cautiverio. Para el inicio de una investigación de una especie silvestre y para la actividad de la acuicultura, se establece criterios técnicos en el manejo, lo que se ha efectuado en esta experiencia, como captura y acondicionamiento, proceso de alimentación y evaluación de los factores fisicoquímicos.

Reyes et al. (2012) señala que los resultados que se obtienen para conformar un plantel de reproductores de bacalao destinados a emprender el cultivo experimental de este importante pez marino (desde la experiencia que se tiene) se puede obtener una sobrevivencia general de $13 \%$ en promedio, y esto en tres campañas de captura, y un período de tres años de mantención de los peces en estanques de $30 \mathrm{~m}^{3}$ en tierra, en un sistema de agua de mar circulante con control de factores ambientales.

Los ejemplares de Anisotremus scapularis capturados del medio natural para formar el plantel de reproductores siguieron un proceso de adaptación a un sistema de cautiverio, donde los resultados fueron de una sobrevivencia de 73.34 $\%$, en promedio de las actividades de captura; siendo muy favorable para las condiciones de cultivo en laboratorio, lo que en otras experiencias de captura de peces marinos reportan menores porcentajes de sobrevivencias.

Por su parte, Aristizabal (2006) menciona que los reproductores de besugo fueron alimentados a saciedad tres veces por semana durante el otoño, invierno y primavera; y durante el verano a una tasa de alimentación del $2 \%$ de la biomasa. El alimento consistió en calamar picado con suplemento de pienso, producido en el laboratorio con altos contenidos de proteína minerales y ácidos grasos. La alimentación en reproductores de Anisotremus scapularis fue ad libitum, y todos los días, al inicio del acondicionamiento se les dio muy muy Emerita analoga y posteriormente se les dio pellet húmedo, elaborado in situ.

Según Boza et al., (2008), en el caso de Pargo manchado, los huevos fertilizados fueron transparentes, esféricos, pelágicos y no adheribles, con un espacio perivitelino muy pequeño, los huevos fertilizados tuvieron un diámetro de $0.857 \mathrm{~mm}$. Los resultados obtenidos en esta experiencia con Anisotremus scapularis es el reporte del primer desove espontáneo, lo que es muy promisorio para la actividad de la acuicultura. Considerando que es una etapa muy crítica en otras especies silvestres, pues no se puede lograr el desove en cautiverio. Los resultados obtenidos en esta experiencia fueron muy alentadores para la especie, con resultados de $79.10 \%$ de viabilidad de los huevos y una eclosión de $93.2 \%$, lo que es altamente satisfactorio en esta etapa.

Ya acondicionados, durante el primer año, los peces presentaron una alta tasa de mortalidad, resultando entre el $3 \%$ y $26 \%$ de sobrevivencia en los estanques (Reyes et al., 2012). La sobrevivencia en reproductores de sargo es un $73.34 \%$.

Después de nueve meses de acondicionamiento de pargo palmero, 2 hembras y 3 machos del fototermoperiodo artificial maduraron gonadalmente (Castaño, 2017). Luego de 7 meses de acondicionamiento los sargos desovaron en forma espontánea.

\section{AGRADECIMIENTO}

A fondos del canon, sobrecanon y regalías mineras de la Universidad Nacional Jorge Basadre Grohmann, por haber permitido desarrollar y seguir con este Proyecto.

\section{REFERENCIA}

ALLEN, G., ROBERTSON, R., RIVERA, F., EDGAR, G. \& MERLEN, G. Anisotremus scapularis. The IUCN Red List of Threatened Species 2010: e. T 183256 A 8081555.2010 . http://dx.doi.org/10.2305/IUCN.UK.201 0-3.RLTS.T183256A8081555.en.

ARISTIZABAL, O. Desove en cautiverio y calidad de los huevos y larvas del besugo, Pagrus pagrus (L.). 24. 2006.

BOZA, J., CALVO E., SOLIS, N., KOMEN, J. Desove inducido y crecimiento larval del pargo manchado, Lutjanus guttatus, en la Estación de Biología Marina de Puntarenas, Costa Rica. Ciencias marinas, 34(2), 239-252, 2008. 
CARRILlO, A.; MUÑOZ, J.; ROCHA, A.; M O L É S, G .; Z A N U Y, S .; FERNÁNDEZ, H. IZQUIERDO. CERDÀ, J. HERRÁEZ, M. GRACIA, V. VALDEBENITO. La reproducción de los peces aspectos básicos y sus aplicaciones en la acuicultura. Madrid. España: Fundación OESA. Crianza de la corvina (Argyrosomus regius), Cuadernos de Acuicultura $\mathrm{N}^{\circ} 03$, Madrid, España: Fundación OESA. 2009.

CASTAÑO, F. (2017). Seguimiento y evaluación de la madurez gonadal de reproductores cautivos de pargo palmero Lutjanus analis (Cuvier, 1828), mediante la medición de calcio y esteroides sexuales en el plasma sanguíneo durante dos fototermoperíodos de acondicionamiento (B.S. thesis). Universidad de Bogotá Jorge Tadeo Lozano.

COLL, J. Actualidad y futuro de la acuicultura española. Revista AquaTIC, (14). 2016.

CHILI V., RODRÍGUEZ L., PINO J. Protocolo de reproducción de lenguado nativo Paralichthys adspersus (Steidachner, 1867), bajo condiciones de laboratorio. Centro de Acuicultura Morro Sama, Sub Dirección de Asistencia Técnica y Transferencia Tecnológica, Fondo Nacional de Desarrollo PesqueroF O N D E P E S. $\quad \begin{array}{llll}2 & 0 & 0 & 9\end{array}$. https://www.fondepes.gob.pe/images/src acui/PROT_REP_LENG_NAT.pdf.

CHIRICHIGNO, N. Clave para identificar los peces marinos del Perú. 1974.

FAO. The state of world fisheries and aquaculture. Fisheries and Aquaculture Department. Rome. 2014.

GALLARDO, P. Antecedentes preliminares del cultivo de bacalao de profundidad (Dissostichus eleginoides; Nototheniidae) en la región de Magallanes, Chile. Anales del Instituto de la Patagonia,44(3): 77-84, 2016. https://doi.org/10.4067/S0718686x2016000300008

GILBERT, C. The living marine resources of the western central ATLANTIC. Copeia, $2005(1), 201-212,2005$. https://doi.org/10.1643/OT-04-309

GONZÁLEZ, F. La acuicultura y el desarrollo económico. Boletín. Instituto Español de Oceanografía, 18(1-4), 265-274, 2011.

LLOYD, M. \& LIVINGSTONE, H. Marine fish farming in Scotland: Proprietorial behaviour and the public interest. Journal of Rural Studies, 7(3), 253-263, 1991. https://doi.org/10.1016/07430167(91)90088-A

MUÑOZ, A., SEGOVIA, E., y FLORES, H. Acondicionamiento de reproductores, desove y cultivo larval de Graus nigra (Philippi, 1887) (Kyphosidae: Girellinae). Latin american journal of aquatic research, 40(SPECISSUE), 584595, 2012. https://doi.org/103864/vol40issue 3-fulltext-8

PAURO J. CHILI V. y RODRÍGUEZ L. Manual de Cultivo de Turbot. Centro de Acuicultura Morro Sama, Sub Dirección de Asistencia Técnica y Transferencia Tecnológica, Fondo Nacional de Desarrollo Pesquero-FONDEPES. 2007.

REYES, A., KIDO, R. y MORENO, C., Captura y mantención de Dissostichus eleginoides para conformar un plantel de reproductores. Latin american journal of aquatic research, 40(4), 1066-1071, 2012. https://doi.org/103856/vol40issue4-fulltext-21

ROCHA, A., LINDEMAN, C., ROCHA, R. y LESSIOS, A. Historical biogeography and speciation in the reef fish genus Haemulon (Teleostei: Haemulidae). Molecular Phylogenetics and Evolution, 48 ( 3 ), $918-928,2008$. https://doi.org/10.1016/j.ympev.2008.05 .024 .

TAVERA. J. Análisis filogenético y biogeográfico del género Anisotremus, Gill 1861(Perciformes: Humalidae). 2006.

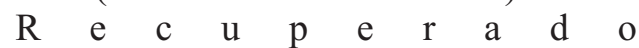
https://www.repositoriodigital.ipn.mx/bi tstream/123456789/15534/1 taveravl.pdf 